\title{
1.55670
}

\section{CLASSIFICATION OF \\ LUNAR SATELLITE ORBITS}

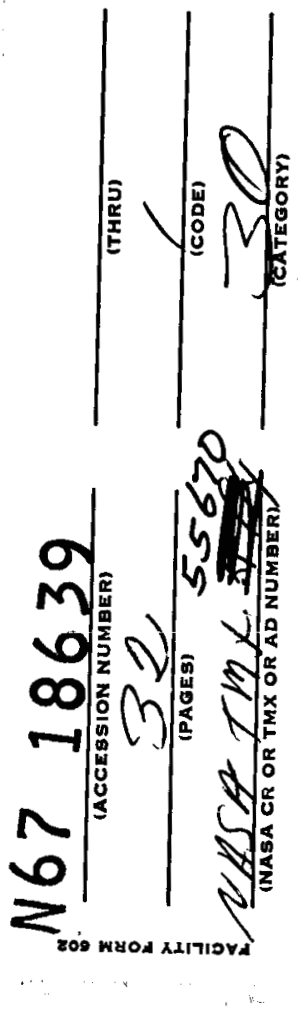

GPO PRICE

s

CFSTI PRICE(S) S

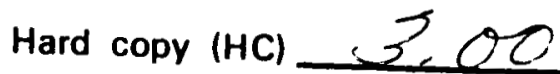

Microfiche (MF)

16.5

B I ff 653 July 65

THEODORE L. FELSENTREGER 


\section{CLASSIFICATION OF LUNAR SATELLITE ORBITS}

by

Theodore L. Felsentreger

August 1966

Goddard Space Flight Center

Greenbelt, Maryland 


\title{
CLASSIFICATION OF LUNAR SATELLITE ORBITS
}

\author{
by
}

Theodore L. Felsentreger

\section{SUMMARY}

A classification is presented of lunar satellite orbits perturbed by the earth and by the non-sphericity of the lunar gravitational field (the second zonal harmonic only). The orbits are arranged in three categories - those in which the argument of perilune circulates, those in which it "librates" about some odd multiple value of $\pi / 2$, and a class of "transition" orbits which belong to neither of the other two classes.

The discussion is kept as general as possible by holding the semi-major axis arbitrary. In addition, two examples are presented to clarify the analysis. Graphs for these cases show the permissible ranges of the two parameters involved (integrals of the satellite motion) for the orbit classes. 
CLASSIFICATION OF LUNAR SATELLITE ORBITS

\section{INTRODUCTION}

Several studies have been made of the long-term effects on artificial satellite orbits disturbed by a third body (references 1, 2, 3 and 4). In addition, Lidov (4) has extended the analysis to include effects caused by the non-sphericity of the gravitational field of the central body (the second zonal harmonic only), in the particular case when the inclination of the disturbing body's orbit plane to the equatorial plane of the central body is $90^{\circ}$. However, this simplification is not applicable when the central body is the moon, the angle in question being about $6^{\circ} 41^{\prime}$.

The orbital motion of an artificial lunar satellite has already been studied by several people, among whom are Kozai (5) and Giacaglia, et al. (6). However, adequate analytical representations for the long-period and secular effects caused by the earth have proved particularly troublesome to obtain - for instance, these two papers present solutions involving elliptic integrals. Later solutions by Frost (7) and Fisher and Felsentreger (8) (which do not involve elliptic integrals) depend upon whether the motion of the argument of perilune is circulatory (secular) or libratory (periodic). It was therefore deemed appropriate to define the regions of circulation and libration.

The disturbing forces which will be considered here, then, give rise to long-period and secular effects caused by the earth and secular perturbations due to the second zonal harmonic of the lunar gravitational field.

Orbits will be divided essentially into two classes - those in which the argument of perilune either circulates or librates. The discussion will be limited to a presentation of the ranges of permissible values for two parameters (integral constants), which of course depend upon initial conditions. Values of the parameters for orbits which fall into neither category ("transition" orbits) lie on the boundaries of the ranges.

A list of symbols appears in Appendix A. 
EQUATIONS OF MOTION AND CONSTANTS OF THE MOTION

The equations of motion for $\eta=\sqrt{1-\mathrm{e}^{2}}$ and the argument of perilune $\mathrm{g}$ are (6)

$$
\begin{gathered}
\frac{\mathrm{d} \eta}{\mathrm{dt}}=\frac{-15}{4} \frac{a_{1}}{\mathrm{q}} \mathrm{n}\left(1-\eta^{2}\right)\left(1-\cos ^{2} \mathrm{i}\right) \sin 2 \mathrm{~g} \\
\frac{\mathrm{dg}}{\mathrm{dt}}=-\frac{3}{2} \frac{\alpha_{1}}{\mathrm{q}} \frac{\mathrm{n}}{\eta}\left[-2 \eta^{2}+5\left(\eta^{2}-\cos ^{2} \mathrm{i}\right) \sin ^{2} \mathrm{~g}\right]-\frac{3}{4} \alpha_{2} \frac{\mathrm{n}}{\eta^{4}}\left(1-5 \cos ^{2} \mathrm{i}\right) .
\end{gathered}
$$

The two integral constants of the motion are readily found to be

$$
\begin{aligned}
& \alpha=\eta^{2} \cos ^{2} \mathrm{i} \\
& \mathrm{c}=\left(1-\eta^{2}\right)\left(1-\frac{5}{2} \sin ^{2} \mathrm{i} \sin ^{2} \mathrm{~g}\right)-\frac{1}{6} \mathrm{~A} \frac{1-3 \cos ^{2} \mathrm{i}}{\eta^{3}},
\end{aligned}
$$

where

$$
A=\frac{\alpha_{2}}{\alpha_{1} / q} .
$$

In the case where the moon's second zonal harmonic $\mathrm{J}_{2}$ is neglected (i.e., $A=0$ ), the parameters become the $a$ and $\beta$ of Lorell (1), who has presented $a$ classification of orbits disturbed only by a third body. However, the analysis is considerably more complicated when $\mathrm{J}_{2}$ is not considered negligible.

To begin the study, it will be assumed that for all orbits the argument of perilune must reach some odd multiple of $\pi / 2$. Therefore, the maximum $c, a$ region compatible with elliptic orbits is that for which $\sin ^{2} \mathrm{~g}=1$ is allowable. If the argument of perilune is to circulate, then $\mathrm{g}$ must eventually become 0 . Hence, the region in the c, a plane describing "circulatory" orbits is that 
for which both $\sin ^{2} \mathrm{~g}=0$ and $\sin ^{2} \mathrm{~g}=1$ are allowable. The non-intersection of the two regions gives "libratory" orbits.

VALUES OF a AND c FOR WHICH $\mathrm{SIN}^{2} \mathrm{~g}=1$

For $\sin ^{2} g=1$, c can be rewritten as

$$
c=\frac{3 \eta^{7}-(3+5 \alpha) \eta^{5}+5 \alpha \eta^{3}-\frac{1}{3} A \eta^{2}+A \alpha}{2 \eta^{5}}
$$

which, for $\eta=1$ (i.e., $\mathrm{e}=0$ ), describes the line

$$
c=-\frac{1}{6} A(1-3 a)
$$

For a particular value of $\eta, \alpha$ achieves its greatest value when $\cos ^{2} i=1$. Replacing $\eta^{2}$ in Equation (3) by $a$, one obtains

$$
c=1-a+\frac{\frac{1}{3} A}{\alpha^{3 / 2}}
$$

which can be rewritten as

$$
c=-\frac{1}{6} \mathrm{~A}(1-3 \alpha)-\frac{\left(\alpha^{1 / 2}-1\right)\left[\left(1+\frac{1}{2} \mathrm{~A}\right) \alpha^{2}+\left(1+\frac{1}{2} \mathrm{~A}\right) \alpha^{3 / 2}+\frac{1}{3} \mathrm{~A} \alpha+\frac{1}{3} \mathrm{~A} \alpha^{1 / 2}+\frac{1}{3} \mathrm{~A}\right]}{a^{3 / 2}} .
$$

The last term in Equation (6) is always $\leq 0$, indicating that Equation (5) describes a curve in the $c, \alpha$ plane to the right of the line $c=-(1 / 6) A(1-3 \alpha)$, and which asymptotically approaches the axis $a=0$. Equations (4) and (5) inter sect at $\alpha=1, c=(1 / 3) A$. Therefore, the region to the right of the line of Equation (4) and bounded by Equation (5) and $\alpha=0$, gives permissible values of $\alpha$ and $c$.

There remains the region to the left of the line to be explored. Equation (3) can be rewritten

$$
\alpha=\frac{\eta^{2}\left[(2 \mathrm{c}+3) \eta^{3}-3 \eta^{5}+\frac{1}{3} \mathrm{~A}\right]}{5\left(\eta^{3}-\eta^{5}\right)+\mathrm{A}}
$$


holding c constant and taking the derivative with respect to $\eta$ yields

$$
\frac{\mathrm{d} \alpha}{\mathrm{d} \eta}=\frac{2 \eta\left[15 \eta^{10}-30 \eta^{8}+5(2 \mathrm{c}+3) \eta^{6}-8 \mathrm{~A} \eta^{5}+5 \mathrm{~A}\left(\mathrm{c}+\frac{4}{3}\right) \eta^{3}+\frac{1}{3} \mathrm{~A}^{2}\right]}{\left[5\left(\eta^{3}-\eta^{5}\right)+\mathrm{A}\right]^{2}} .
$$

It is desirable to describe the roots of

$$
\mathrm{f}(\eta)=15 \eta^{10}-30 \eta^{8}+5(2 \mathrm{c}+3) \eta^{6}-8 \mathrm{~A} \eta^{5}+5 \mathrm{~A}\left(\mathrm{c}+\frac{4}{3}\right) \eta^{3}+\frac{1}{3} \mathrm{~A}^{2}
$$

in order to determine possible maximum values of $a$.

Now, $a=0$ at $\eta=0$. Since $f(0)=(1 / 3) A^{2}>0$, $a$ increases from 0 until $\eta$ assumes the value of a root of $f(\eta)$. Suppose, for the moment, that $\eta_{1}$ is a root of $\mathrm{f}(\eta)$. If it can be shown that $\alpha(1) \leq \alpha\left(\eta_{1}\right)$, then one can conclude that $\alpha$ attains a maximum value either at $\eta_{1}$ or at some other root of $f(\eta)$ in $0<\eta \leq 1$.

From Equation (9), then,

$$
c=\frac{-15 \eta_{1}^{10}+30 \eta_{1}^{8}-15 \eta_{1}^{6}+8 \mathrm{~A} \eta_{1}^{5}-\frac{20}{3} \mathrm{~A} \eta_{1}^{3}-\frac{1}{3} \mathrm{~A}^{2}}{5 \eta_{1}^{3}\left(2 \eta_{1}^{3}+\mathrm{A}\right)}
$$

Substitution of Equation (10) into Equation (7) yields ( for $\eta=\eta_{1}$ )

$$
\alpha\left(\eta_{1}\right)=\frac{\eta_{1}^{2}\left[-30 \eta_{1}^{10}+30 \eta_{1}^{8}+\mathrm{A} \eta_{1}^{5}+5 \mathrm{~A} \eta_{1}^{3}+\mathrm{A}^{2}\right]}{5\left[5\left(\eta_{1}^{3}-\eta_{1}^{5}\right)+\mathrm{A}\right]\left(2 \eta_{1}^{3}+\mathrm{A}\right)}
$$

and, for $\eta=1$,

$$
a(1)=\frac{-30 \eta_{1}^{10}+60 \eta_{1}^{8}-\frac{10}{3}(9-\mathrm{A}) \eta_{1}^{6}+16 \mathrm{~A} \eta_{1}^{5}-\frac{5}{3} \mathrm{~A}(8-\mathrm{A}) \eta_{1}^{3}-\frac{2}{3} \mathrm{~A}^{2}}{5 \mathrm{~A} \eta_{1}^{3}\left(2 \eta_{1}^{3}+\mathrm{A}\right)} .
$$


Then,

$$
\alpha(1)-\alpha\left(\eta_{1}\right)=\frac{\left(\eta_{1}-1\right)^{2} \mathrm{~g}\left(\eta_{1}\right)}{15 \mathrm{~A} \eta_{1}^{3}\left(2 \eta_{1}^{3}+\mathrm{A}\right)\left[5\left(\eta_{1}^{3}-\eta_{1}^{5}\right)+\mathrm{A}\right]},
$$

where

$$
\begin{aligned}
\mathrm{g}\left(\eta_{1}\right) & =90(5+\mathrm{A}) \eta_{1}^{13}+180(5+\mathrm{A}) \eta_{1}^{12}+180 \mathrm{~A} \eta_{1}^{11}-180(5-\mathrm{A}) \eta_{1}^{10}-10(45-13 \mathrm{~A}) \eta_{1}^{9} \\
& -\mathrm{A}(250+3 \mathrm{~A}) \eta_{1}^{8}-2 \mathrm{~A}(290+3 \mathrm{~A}) \eta_{1}^{7}-\mathrm{A}(290+49 \mathrm{~A}) \eta_{1}^{6}-92 \mathrm{~A}^{2} \eta_{1}^{5}-100 \mathrm{~A}^{2} \eta_{1}^{4} \\
& -\mathrm{A}^{2}(50+3 \mathrm{~A}) \eta_{1}^{3}-6 \mathrm{~A}^{3} \eta_{1}^{2}-4 \mathrm{~A}^{3} \eta_{1}-2 \mathrm{~A}^{3}
\end{aligned}
$$

The Theorem of Vincent and Descartes' Rule of Signs establishes that $\mathrm{g}\left(\eta_{1}\right)$ has no root in $0<\eta_{1}<1$ (see Appendix B). Since $\mathrm{g}(0)=-2 \mathrm{~A}^{3}<0$ and $\mathrm{g}(1)=$ $-15 A\left(A^{2}+20 A+24\right)<0$, one may conclude that

$$
a(1)-a\left(\eta_{1}\right) \leq 0
$$

for $0<\eta_{1} \leq 1$; equality occurs only when $\eta_{1}=1$.

It must now be shown that $a\left(\eta_{1}\right) \leq 1$ for any $\operatorname{root} \eta_{1}$ of $f(\eta)$. From Equation (11),

$$
\begin{aligned}
a\left(\eta_{1}\right) \leq 1 \Longleftrightarrow \mathrm{h}\left(\eta_{1}\right)= & -30 \eta_{1}^{12}+30 \eta_{1}^{10}+50 \eta_{1}^{8}+\mathrm{A} \eta_{1}^{7}-50 \eta_{1}^{6} \\
& +30 \mathrm{~A} \eta_{1}^{5}-35 \mathrm{~A} \eta_{1}^{3}+\mathrm{A}^{2} \eta_{1}^{2}-5 \mathrm{~A}^{2} \leq 0 .
\end{aligned}
$$

Again, it can be shown that $h\left(\eta_{1}\right)$ has no root in $0 \leq \eta_{1} \leq 1$ (see Appendix B). Since $h(0)=-5 \mathrm{~A}^{2}<0$, it has been established that $a\left(\eta_{1}\right)<1$ for any root $\eta_{1}$ of $\mathrm{f}(\eta)$. 
Finally, it must be demonstrated that, for any value of $c$ less than $-(1 / 6) A(1-3 \alpha), f(\eta)$ has at least one root. From Equation (9), $f(0)=(1 / 3) A^{2}>0$. Existence of a root between 0 and 1 is assured if $f(1)<0$ - hence

$$
\mathrm{f}(1)<0 \Longleftrightarrow \mathrm{c}<\frac{\mathrm{A}(4-\mathrm{A})}{15(2+\mathrm{A})} \text {. }
$$

However, when $c=A(4-A) / 15(2+A), \eta=1$ is a root of $f(\eta)$, and the point $a=$ $(6+A) / 5(2+A), c=A(4-A) / 15(2+A)$ lies on the line $c=-(1 / 6) A(1-3 a)$. Thus, $\mathrm{c}<\mathrm{A}(4-\mathrm{A}) / 15(2+\mathrm{A}) \Longrightarrow \mathrm{f}(\eta)$ has at least one $\operatorname{root} \eta_{1}$ in $0<\eta_{1}<1$. In addition, since $a(1)<\alpha\left(\eta_{1}\right)$ for $\eta_{1} \neq 1$, the point $a\left(\eta_{1}\right)$, c lies above the line $\mathbf{c}=$ $-(1 / 6)$ A $(1-3 \alpha)$.

Hence, the curve whose parametric equations are (for $0<\eta_{1} \leq 1$ )

$$
\begin{aligned}
& \mathrm{c}\left(\eta_{1}\right)=\frac{-15 \eta_{1}^{10}+30 \eta_{1}^{8}-15 \eta_{1}^{6}+8 \mathrm{~A} \eta_{1}^{5}-\frac{20}{3} \mathrm{~A} \eta_{1}^{3}-\frac{1}{3} \mathrm{~A}^{2}}{5 \eta_{1}^{3}\left(2 \eta_{1}^{3}+\mathrm{A}\right)} \\
& a\left(\eta_{1}\right)=\frac{\eta_{1}^{2}\left(-30 \eta_{1}^{10}+30 \eta_{1}^{8}+\mathrm{A} \eta_{1}^{5}+5 \mathrm{~A} \eta_{1}^{3}+\mathrm{A}^{2}\right)}{5\left[5\left(\eta_{1}^{3}-\eta_{1}^{5}\right)+\mathrm{A}\right]\left(2 \eta_{1}^{3}+\mathrm{A}\right)}
\end{aligned}
$$

represents the upper boundary to the permissible $c$, a region lying to the left of the line $\mathrm{c}=-(1 / 6) \mathrm{A}(1-3 a)$. It intersects this line at the point $\mathrm{c}=$ $\mathrm{A}(4-\mathrm{A}) / 15(2+\mathrm{A}), \alpha=(6+\mathrm{A}) / 5(2+\mathrm{A})$, and approaches the $\alpha=0$ axis asymptotically.

$$
\text { VALUES OF a AND c FOR WHICH } \mathrm{SIN}^{2} \mathrm{~g}=0
$$

For $\sin ^{2} g=0$, Equation (2) becomes

$$
c=\frac{-\eta^{7}+\eta^{5}-\frac{1}{6} \mathbf{A} \eta^{2}+\frac{1}{2} \mathbf{A} \alpha}{\eta^{5}},
$$

which, for $\eta=1$, again becomes the line $c=-(1 / 6) \mathrm{A}(1-3 a)$. In addition, for $\cos ^{2} i=1$, Equation (16) becomes

$$
c=1-\alpha+\frac{\frac{1}{3} A}{\alpha^{3 / 2}},
$$


which describes the same curve as for $\sin ^{2} g=1$. Therefore, the permissible region to the right of $c=-(1 / 6) A(1-3 \alpha)$ is the same as for $\sin ^{2} g=1$.

Now, Equation (16) can be rewritten as

$$
\alpha=\frac{2}{\mathrm{~A}} \eta^{2}\left[\eta^{5}+(\mathrm{c}-1) \eta^{3}+\frac{1}{6} \mathrm{~A}\right]
$$

whose derivative with respect to $\eta$ (for constant c) is

$$
\frac{\mathrm{d} \alpha}{\mathrm{d} \eta}=\frac{2}{\mathrm{~A}} \eta\left[7 \eta^{5}+5(\mathrm{c}-1) \eta^{3}+\frac{1}{3} \mathrm{~A}\right] .
$$

Thus, an analysis of the roots of

$$
\mathrm{F}(\eta)=7 \eta^{5}+5(\mathrm{c}-1) \eta^{3}+\frac{1}{3} \mathrm{~A}
$$

is in order.

Obvioüly, since $F(0)=(1 / 3) A>0$, a increases from a value of 0 at $\eta=0$ until a root of $F(\eta)$ is reached, assuring the existence of a relative maximum for a in $0<\eta \leq 1$ (providing $F(\eta)$ has a root). Hence, assuming $\eta_{1}$ to be a root of $F(\eta)$, it again behooves one to show that $\alpha(1) \leq \alpha\left(\eta_{1}\right)$.

When $\eta=\eta_{1}$ is a root of $\mathrm{F}(\eta)$, Equation (19) gives

$$
c=\frac{-7 \eta_{1}^{5}+5 \eta_{1}^{3}-\frac{1}{3} \mathrm{~A}}{5 \eta_{1}^{3}}
$$

Equation (17) then becomes (for $\eta=\eta_{1}$ )

$$
\alpha\left(\eta_{1}\right)=\frac{\eta_{1}^{2}\left(-4 \eta_{1}^{5}+\mathrm{A}\right)}{5 \mathrm{~A}}
$$


for $\eta=1$,

$$
a(1)=\frac{-42 \eta_{1}^{5}+5(6+\mathrm{A}) \eta_{1}^{3}-2 \mathrm{~A}}{15 \mathrm{~A} \eta_{1}^{3}}
$$

Then,

$$
a(1)-a\left(\eta_{1}\right)=\frac{\left(\eta_{1}-1\right)^{2} \mathrm{G}\left(\eta_{1}\right)}{15 \mathrm{~A} \eta_{1}^{3}},
$$

where

$$
\mathrm{G}\left(\eta_{1}\right)=12 \eta_{1}^{8}+24 \eta_{1}^{7}+36 \eta_{1}^{6}+48 \eta_{1}^{5}+60 \eta_{1}^{4}+3(10-\mathrm{A}) \eta_{1}^{3}-6 \mathrm{~A} \eta_{1}^{2}-4 \mathrm{~A} \eta_{1}-2 \mathrm{~A} .
$$

$\mathrm{G}\left(\eta_{1}\right)$ has exactly one positive root. For A $<14$, this root is in $0<\eta_{1}<1$; for $\mathrm{A}=14$, the root is $\eta_{1}=1$; and for $\mathrm{A}>14, \mathrm{G}\left(\eta_{1}\right)$ has no roots in $0<\eta_{1} \leq 1$ (see Appendix B). Therefore, $a(1)-\alpha\left(\eta_{1}\right) \leq 0$ for $\mathrm{A} \geq 14$; for $\mathrm{A}<14$, designating the root of $\mathrm{G}\left(\eta_{1}\right)$ by $\eta_{1}^{*}$,

$$
a(1)-a\left(\eta_{1}\right)=\frac{\left(\eta_{1}-1\right)^{2}\left(\eta_{1}-\eta_{1}^{*}\right) \mathbf{G}^{*}\left(\eta_{1}\right)}{15 \mathrm{~A} \eta_{1}^{3}},
$$

where $\mathrm{G}^{*}\left(\eta_{1}\right)>0$. Thus, $a(1)-a\left(\eta_{1}\right) \leq 0$ when $\eta_{1} \leq \eta_{1}^{*}$ - equality occurs (for A $<14$ ) only when $\eta_{1}=\eta_{1}^{*}$.

The foregoing has also shown that the point $c, a\left(\eta_{1}\right)$ lies above the line $\mathrm{c}=-(1 / 6) \mathrm{A}(1-3 \alpha)$ when $0<\eta_{1}<1$ (for $\left.\mathrm{A} \geq 14\right)$, and when $0<\eta_{1}<\eta_{1}^{*}($ for $\mathrm{A}<14)$. For the two cases respectively, the points

$-c=-\frac{1}{5}\left(2+\frac{1}{3} A\right), a=\frac{-4+A}{5 A}$ and $c=\frac{-7 \eta_{1}^{* 5}+5 \eta_{1}^{* 3}-\frac{1}{3} A}{5 \eta_{1}^{* 3}}, a=\frac{\eta_{1}^{* 2}\left(-4 \eta_{1}^{* 5}+A\right)}{5 A}$

are on the line. 
As before, now, it must be shown that $\alpha\left(\eta_{1}\right) \leq 1$ for any root $\eta_{1}$ of $F(\eta)$. From Equation (21),

$$
\alpha\left(\eta_{1}\right) \leq 1 \Longleftrightarrow \mathrm{H}\left(\eta_{1}\right)=-4 \eta_{1}^{7}+\mathrm{A} \eta_{1}^{2}-5 \mathrm{~A} \leq 0 .
$$

But $\mathrm{H}\left(\eta_{1}\right)$ has no root in $0<\eta_{1} \leq 1$ (see Appendix B). Since $H(0)=-5 \mathrm{~A}<0$, $\alpha\left(\eta_{1}\right)<1$ for any root $\eta_{1}$ of $\mathrm{F}(\eta)$.

Finally, in the case where $A \geq 14$,

$$
c \leq-\frac{1}{5}\left(2+\frac{1}{3} A\right) \Longrightarrow F(1)=5 c+2+\frac{1}{3} A \leq 0
$$

Also, $F(0)=(1 / 3) A>0$, so $F(\eta)$ has at least one root in $0<\eta \leq 1$. The existence of at least one root of $F(\eta)$ in the case where $A<14$ will not be proved here however, it can be proved after choosing a value of $A<14$.

To summarize, then, the curve whose parametric equations are

$$
\begin{aligned}
& c\left(\eta_{1}\right)=\frac{-7 \eta_{1}^{5}+5 \eta_{1}^{3}-\frac{1}{3} A}{5 \eta_{1}^{3}} \\
& a\left(\eta_{1}\right)=\frac{\eta_{1}^{2}\left(-4 \eta_{1}^{5}+A\right)}{5 A}
\end{aligned}
$$

represents the upper boundary (for $\sin ^{2} \mathrm{~g}=0$ ) to the permissible $\mathrm{c}$, a region lying to the left of the line $c=-(1 / 6) A(1-3 \alpha)$. When $A \geq 14$, the parameter $\eta_{1}$ may take on all values in $0<\eta_{1} \leq 1$. However, when $A<14, \eta_{1}$ is restricted to the range $0<\eta_{1} \leq \eta_{1}^{*}<1$, where $\eta_{1}^{*}$ is the root of $\mathrm{G}\left(\eta_{1}\right)$ defined by Equation (24).

Figure 1 depicts the $c$, a regions defined in the previous two sections. 


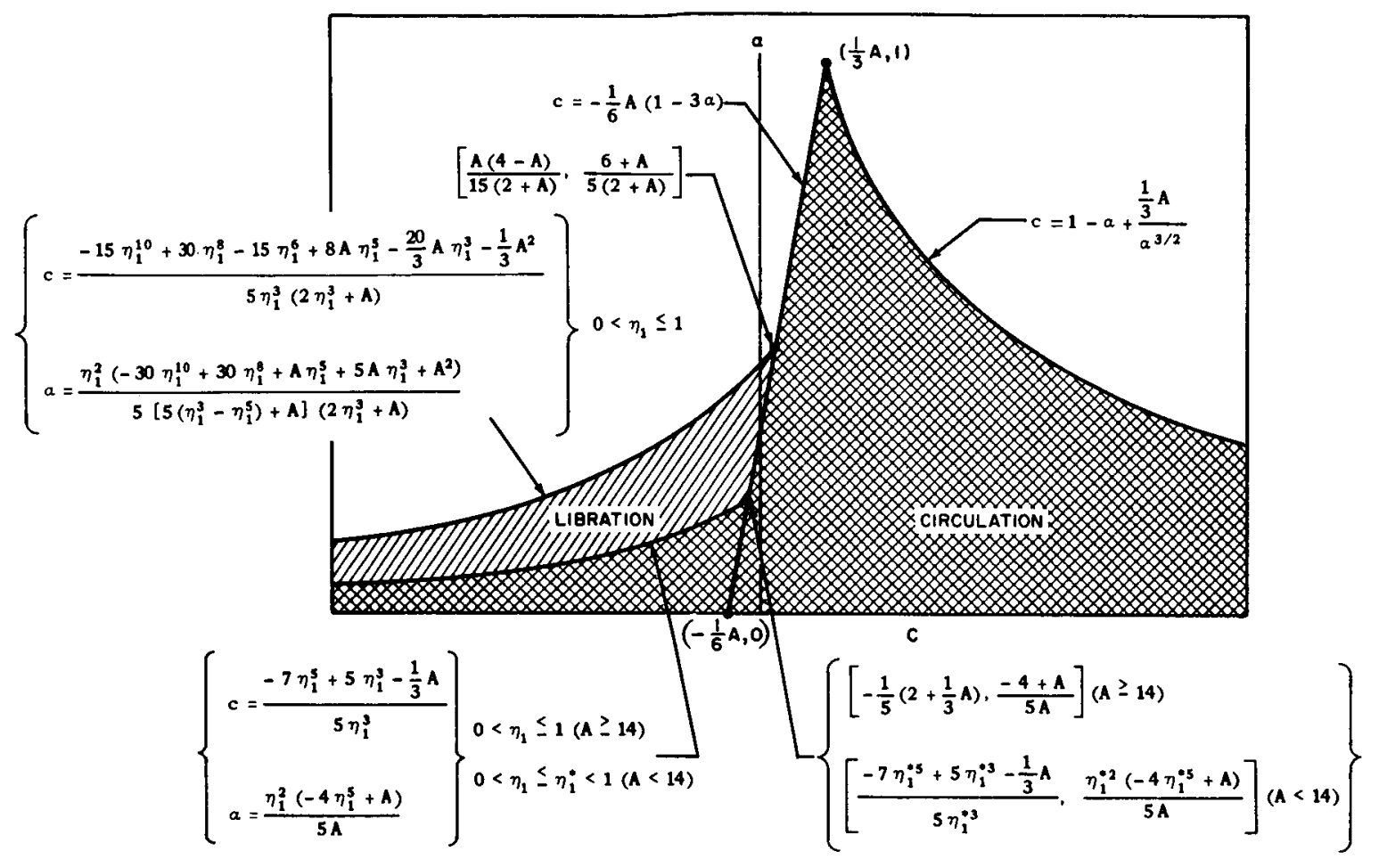

Figure 1

\section{EXAMPLES}

The two cases to be presented here correspond to values for the semimajor axis of the satellite's orbit of 2 and 7.4822577 moon radii. Then, the values for A are 164.97081 and .22510948, respectively. For the first case, Equations (4), (5), (15), and (25) become

$$
\begin{aligned}
& c=-27.495135(1-3 \alpha) \\
& c=1-\alpha+\frac{54 \cdot 990270}{\alpha^{3 / 2}}
\end{aligned}
$$




$$
\begin{array}{ll}
c=\frac{-15 \eta_{1}^{10}+30 \eta_{1}^{8}-15 \eta_{1}^{6}+1319.7665 \eta_{1}^{5}-1099.8054 \eta_{1}^{3}-9071.7893}{5 \eta_{1}^{3}\left(2 \eta_{1}^{3}+164.97081\right)} & \\
a\left(\eta_{1}\right)=\frac{\eta_{1}^{2}\left(-30 \eta_{1}^{10}+30 \eta_{1}^{8}+164.97081 \eta_{1}^{5}+824.85405 \eta_{1}^{3}+27215.368\right)}{5\left[5\left(\eta_{1}^{3}-\eta_{1}^{5}\right)+164.97081\right]}\left(2 \eta_{1}^{3}+164.97081\right) & \left(0<\eta_{1} \leq 1\right) \\
c\left(\eta_{1}\right)=\frac{-7 \eta_{1}^{5}+5 \eta_{1}^{3}-54.990270}{5 \eta_{1}^{3}} & \left(0<\eta_{1} \leq 1\right) \\
c\left(\eta_{1}\right)=\frac{\eta_{1}^{2}\left(-4 \eta_{1}^{5}+164.97081\right)}{824.85405} & \quad(2)
\end{array}
$$

For $\mathrm{A}=.22510948$, the root of $\mathrm{G}\left(\eta_{1}\right)$ is $\eta_{1}^{*}=.25110445$; therefore, the equations defining the boundaries of the circulation and libration regions are

$$
\begin{aligned}
& c=-.037518247(1-3 \alpha) \\
& c=1-a+\frac{.075036494}{a^{3 / 2}} \\
& c\left(\eta_{1}\right)=\frac{-15 \eta_{1}^{10}+30 \eta_{1}^{8}-15 \eta_{1}^{6}+1.80087584 \eta_{1}^{5}-1.50072988 \eta_{1}^{3}-.01689143}{5 \eta_{1}^{3}\left(2 \eta_{1}^{3}+.22510948\right)} \\
& a\left(\eta_{1}\right)=\frac{\eta_{1}^{2}\left(-30 \eta_{1}^{10}+30 \eta_{1}^{8}+.22510948 \eta_{1}^{5}+1.12554740 \eta_{1}^{3}+.05067428\right)}{5\left[5\left(\eta_{1}^{3}-\eta_{1}^{5}\right)+.22510948\right]\left(2 \eta_{1}^{3}+.22510948\right)} \\
& c\left(\eta_{1}\right)=\frac{-7 \eta_{1}^{5}+5 \eta_{1}^{3}+.075036494}{5 \eta_{1}^{3}} \\
& \mathrm{a}=\frac{\eta_{1}^{2}\left(-4 \eta_{1}^{5}+.22510948\right)}{1.1255474} \quad\left(0<\eta_{1} \leq 1\right)
\end{aligned}
$$


Tables 1,2, and 3(Appendix C) list values of $\mathrm{c}$ and a for points on the curves defined by the preceding equations. Figures 2, 3, 4, and 5 (Appendix D) show the circulation and libration regions for the two cases. It is readily apparent that the libration region for $a=2 R_{c}$ is considerably smaller than that for the case $a=7.4822577 R_{c}$ - this is a consequence of the fact that the part of the disturbing function dependent upon $\mathrm{J}_{2}$ (which is purely secular) is more significant for smaller values of a .

\section{CONCLUSIONS}

Given a set of initial conditions for an artificial lunar satellite, it can be determined from a graph similar to Figure 1 whether the orbit falls into the circulation case or libration case. If circulation, the method outlined by Frost (7) can be used to compute the long-period and secular effects caused by the earth and the secular effects due to the moon's second zonal harmonic. On the other hand, if the orbit is libratory, the method presented by Fisher and Felsentreger (8) may be employed.

\section{REFERENCES}

1. Lorell, J., "Long Term Behavior of Artificial Satellite Orbits Due to ThirdBody Perturbations," Journal of the Astronautical Sciences, Vol. XII, No. 4, pp. 142-149, Winter, 1965.

2. Williams, R. R., "Long-Term Behaviour of Artificial Satelíite Orbits Perturbed by a Third Body," Space Programs Summary No. 37-23, Vol. IV, Jet Propulsion Laboratory, October 31, 1963.

3. Lidov, M. L., "Evolution of the Orbits of Artificial Satellites of Planets as Affected by Gravitational Perturbations from External Bodies," Russian Supplement AIAAJ No. 8, pp. 1985-2002, August, 1963.

4. Lidov, M. L., "On the Approximated Analysis of the Orbit Evaluation of Artificial Satellites," IUTAM Symposium, Paris, 1962. Proceedings published as Dynamics of Satellites, pp. 168-179, Academic Press, N.Y., 1963.

5. Kozai, Y., "The Motion of a Lunar Orbiter," Journal of the Astronomical Society of Japan, Vol. 15, pp. 301-312, 1963.

6. Giacaglia, G. E. O., Murphy, J. P., and Felsentreger, T. L., in collaboration with Standish, E. M., Jr., and Velez, C. E., "The Motion of a Satellite of the Moon," GSFC X-547-65-218, June, 1965. 
7. Frost, F. A., "Long-Term Motion of a Lunar Satellite," GSFC X-547-65-388, October, 1965.

8. Fisher, D., and Felsentreger, T. L., "The Libration of a Lunar Satellite," GSFC X-547-66-238, May, 1966. 


\section{APPENDIX A}

\section{SYMBOLS}

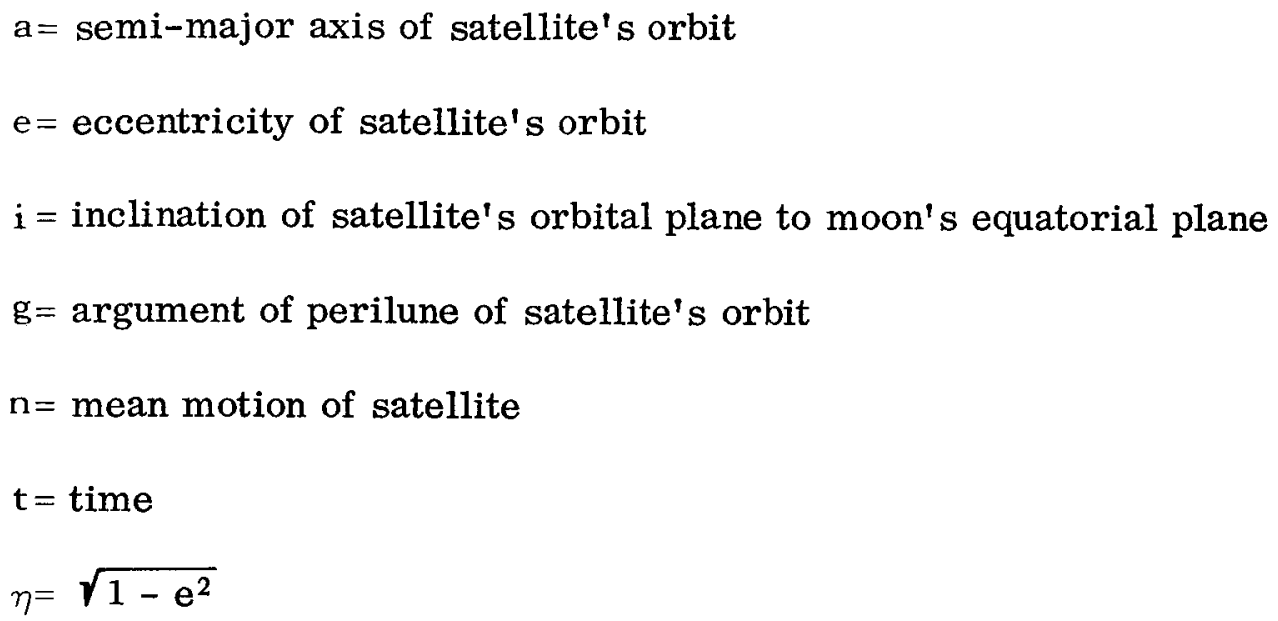

$\alpha, \mathrm{c}=$ constants of the motion 


\section{APPENDIX B}

1. Proof that $\mathrm{g}\left(\eta_{1}\right)$ has no root in $0<\eta_{1}<1$

From Equation (14),

$$
\begin{aligned}
\mathrm{g}\left(\eta_{1}\right)=90 & (5+\mathrm{A}) \eta_{1}^{13}+180(5+\mathrm{A}) \eta_{1}^{12}+180 \mathrm{~A} \eta_{1}^{11}-180(5-\mathrm{A}) \eta_{1}^{10}-10(45-13 \mathrm{~A}) \eta_{1}^{9} \\
& -\mathrm{A}(250+3 \mathrm{~A}) \eta_{1}^{8}-2 \mathrm{~A}(290+3 \mathrm{~A}) \eta_{1}^{7}-\mathrm{A}(290+49 \mathrm{~A}) \eta_{1}^{6}-92 \mathrm{~A}^{2} \eta_{1}^{5}-100 \mathrm{~A}^{2} \eta_{1}^{4} \\
& -\mathrm{A}^{2}(50+3 \mathrm{~A}) \eta_{1}^{3}-6 \mathrm{~A}^{3} \eta_{1}^{2}-4 \mathrm{~A}^{3} \eta_{1}-2 \mathrm{~A}^{3}
\end{aligned}
$$

which, by Descartes' Rule of Signs, has either one positive root, or possibly three (in the case $45 / 13<\mathrm{A}<5$ ). Replacing $\eta_{1}$ by $1 / 1+X(X>0)$ transforms the equation into one having exactly the same number of positive roots as those of $\mathrm{g}\left(\eta_{1}\right)$ in $0<\eta_{1}<1$. Thus, solving $\mathrm{g}\left(\eta_{1}\right)=0$ for $0<\eta_{1}<1$ is equivalent to solving the following equation for $X>0$ (the coefficients of $X^{13}, X^{12}, \ldots X^{5}$ are all obviously negative, so there is no need to write them down explicitly):

$$
\begin{aligned}
0= & a_{13} X^{13}+a_{12} X^{12}+a_{11} X^{11}+a_{10} X^{10}+a_{9} X^{9}+a_{8} X^{8}+a_{7} X^{7}+a_{6} X^{6}+a_{5} X^{5} \\
& -10\left(45+1997 A+3136 A^{2}+602 A^{3}\right) X^{4}-3\left(900+7850 A+7139 A^{2}+934 A^{3}\right) X^{3} \\
& -5\left(1080+3158 A+1915 A^{2}+177 A^{3}\right) X^{2}-10\left(360+516 A+253 A^{2}+17 A^{3}\right) X \\
& -15 A\left(24+20 A+A^{2}\right),
\end{aligned}
$$

where $a_{13}, a_{12}, \ldots, a_{5}<0$. This equation obviously can have no positive solutions - hence, g $\left(\eta_{1}\right)$ has no roots in $0<\eta_{1}<1$.

2. Proof that $\mathrm{h}\left(\eta_{1}\right)$ has no root in $0 \leq \eta_{1} \leq 1$

$$
\mathrm{h}\left(\eta_{1}\right)=-30 \eta_{1}^{12}+30 \eta_{1}^{10}+50 \eta_{1}^{8}+\mathrm{A} \eta_{1}^{7}-50 \eta_{1}^{6}+30 \mathrm{~A} \eta_{1}^{5}-35 \mathrm{~A} \eta_{1}^{3}+\mathrm{A}^{2} \eta_{1}^{2}-5 \mathrm{~A}^{2}
$$


Since $h(0)=-5 A^{2}<0$ and $h(1)=-4 A(1+A)<0$, it remains to be shown that $\mathrm{h}\left(\eta_{1}\right)$ has no roots in $0<\eta_{1}<1$. Equivalently, it must be shown that the transformed equation, after replacing $\eta_{1}$ by $1 / 1+X(X>0)$, has no positive roots. The transformed equation is

$$
\begin{aligned}
0= & -5 A^{2} X^{12}-60 A^{2} X^{11}-329 A^{2} X^{10}-5 A(7+218 A) X^{9}-45 A(7+54 A) X^{8} \\
& -30 A(41+128 A) X^{7}-10\left(5+273 A+441 A^{2}\right) X^{6}-\left(300+3779 A+3708 A^{2}\right) X^{5} \\
& -5\left(140+671 A+453 A^{2}\right) X^{4}-20\left(40+94 A+49 A^{2}\right) X^{3}-5\left(84+124 A+57 A^{2}\right) X^{2} \\
& -10\left(4+10 A+5 A^{2}\right) X-4 A(1+A),
\end{aligned}
$$

which has no non-negative solutions. Consequently, h $\left(\eta_{1}\right)$ has no roots in $0 \leq \eta_{1} \leq 1$

3. Roots of $\mathrm{G}\left(\eta_{1}\right)$ in $0<\eta_{1} \leq 1$

$$
\mathrm{G}\left(\eta_{1}\right)=12 \eta_{1}^{8}+24 \eta_{1}^{7}+36 \eta_{1}^{6}+48 \eta_{1}^{5}+60 \eta_{1}^{4}+3(10-\mathrm{A}) \eta_{1}^{3}-6 \mathrm{~A} \eta_{1}^{2}-4 \mathrm{~A} \eta_{1}-2 \mathrm{~A} .
$$

Finding the roots of $\mathrm{G}\left(\eta_{1}\right)$ in $0<\eta_{1} \leq 1$ is equivalent to finding the positive roots of

$$
\begin{aligned}
& b_{8} X^{8}+b_{7} X^{7}+b_{6} X^{6}+5(1-47 A) X^{5}+35(6-11 A) X^{4}+6(98-67 A) X^{3} \\
& +20(42-13 A) X^{2}+5(126-19 A) X+15(14-A),
\end{aligned}
$$

where $b_{8}, b_{7}, b_{6}<0$ and $\eta_{1}=1 / 1+X$. Descartes' Rule of Signs establishes that the polynomial in $X$ has exactly one positive root for $A \leq 14$, and no positive roots when $A>14$. Consequently, $G\left(\eta_{1}\right)$ has exactly one root in $0<\eta_{1} \leq 1$ for $A \leq 14$, and no roots in $0<\eta_{1} \leq 1$ when $A>14$. Since $G(1)=15(14-A)$, the root is 1 when $\mathrm{A}=14$, and is in $0<\eta_{1}<1$ when $\mathrm{A}<14$. 
4. Proof that $\mathrm{H}\left(\eta_{1}\right)$ has no root in $0<\eta_{1} \leq 1$

$$
\mathrm{H}\left(\eta_{1}\right)=-4 \eta_{1}^{7}+\mathrm{A} \eta_{1}^{2}-5 \mathrm{~A}
$$

$$
H(1)=-5 A<0 \text {, so } 1 \text { is not a root. }
$$

Setting $\eta_{1}=1 / 1+X$, the polynomial set equal to zero is

$$
0=-5 A X^{7}-35 A X^{6}-104 A X^{5}-170 A X^{4}-165 A X^{3}-95 A X^{2}-30 A X-4(1+A)
$$

which obviously has no positive roots. Therefore, $\mathrm{H}\left(\eta_{1}\right)$ has no roots in $0<\eta_{1}<1$. 
PRECEDING PAGE BLANK NOT FILMED.

APPENDIX C

Tables 
Table 1

c, $a$ values for $c=1-a+\frac{\frac{1}{3} A}{a^{3 / 2}}$

$\underline{a}$

c $(A=164.97081)$

c $(A=.22510948)$

. 1

$\mathbf{5 4 . 9 9 0 2 7 0}$

.07503649

.9409

60.310987

.14131618

.8836

66.323153

.20674184

.8281

73.144811

.27147455

.7744

80.918967

.33570943

.7225

89.819971

.39968440

.6724

100.061843

.46369149

.6241

111.909208

.52809180

.5776

125.619822

.59333530

.5329

141.824080

.65998744

.4900

160.831490

.72876529

.4489

183.386990

.80058712

.4096

210.361630

.87664150

.3721

242.896060

.95848490

.3364

282.503110

1.04818164

.3025

331.217530

1.14850822

.2704

391.818790

1.26325735

.2401

468.169490

1.39769967

.2116

565.741440

1.55930176

.1849

1.75887216

.1600

2.01244520

.1369

2.34448300

.1156

2.79353121

.0961

3.42266381

.0784

4.33980761 
Table 2

, a values for $\mathrm{c}=\frac{-15 \eta_{1}^{10}+30 \eta_{1}^{8}-15 \eta_{1}^{6}+8 \mathrm{~A} \eta_{1}^{5}-\frac{20}{3} \mathrm{~A} \eta_{1}^{3}-\frac{1}{3} \mathrm{~A}^{2}}{5 \eta_{1}^{3}\left(2 \eta_{1}^{3}+\mathrm{A}\right)}$

$\alpha=\frac{\eta_{1}^{2}\left(-30 \eta_{1}^{10}+30 \eta_{1}^{8}+\mathrm{A} \eta_{1}^{5}+5 \mathrm{~A} \eta_{1}^{3}+\mathrm{A}^{2}\right)}{5\left[5\left(\eta_{1}^{3}-\eta_{1}^{5}\right)+\mathrm{A}\right]\left(2 \eta_{1}^{3}+\mathrm{A}\right)}$

$\underline{\eta_{1}} \quad \underline{c}(\mathrm{~A}=164.97081) \quad \alpha(\mathrm{A}=164.97081) \quad c(\mathrm{~A}=.22510948) \quad a(\mathrm{~A}=.22510948)$

$1-\quad 10.60285 \quad .20479126$

.02545983

.55953289

$.95-12.58625$

.18367060

$-.00179364$

.45293847

$.90-14.99181$

.16402945

$-.05465415$

.36267636

$.85-17.95302$

.14574676

$-.12887024$

.28707338

$.80 \quad-\quad 21.65669$

.12872644

$-.22039516$

.22453776

$.75 \quad-\quad 26.36940$

.11289356

- .32639709

.17250927

$.70 \quad-\quad 32.48019$

.09819074

$-.44028987$

.13267987

$.65-40.57145$

.08457501

- .56180739

.10053275

.60

.07201504

$-.68716695$

.07578677

$.55-66.82003$

.06048873

- .81440876

.05716197

$.50 \quad-88.78450$

.04998109

- .94307939

.04342257

.45

- 121.56804

.04048246

$-1.07559004$

.03340831

.40

$-172.78868$

.03198710

$-1.21995835$

.02596810

$.35 \quad-257.51846$

.02449195

$-1.39568007$

.02022638

.30

- 408.39139

.01799570

$-1.64784077$

.01545777

$.25 \quad-704.97551$

.01249808

$-2.08708494$

.01126196

.20

$-1375.89282$

.00799932

$-3.02826351$

.00753283 
Table 3

$c, a$ values for $c=\frac{-7 \eta_{1}^{5}+5 \eta_{1}^{3}-\frac{1}{3} \mathrm{~A}}{5 \eta_{1}^{3}}, \alpha=\frac{\eta_{1}^{2}\left(-4 \eta_{1}^{5}+\mathrm{A}\right)}{5 \mathrm{~A}}$

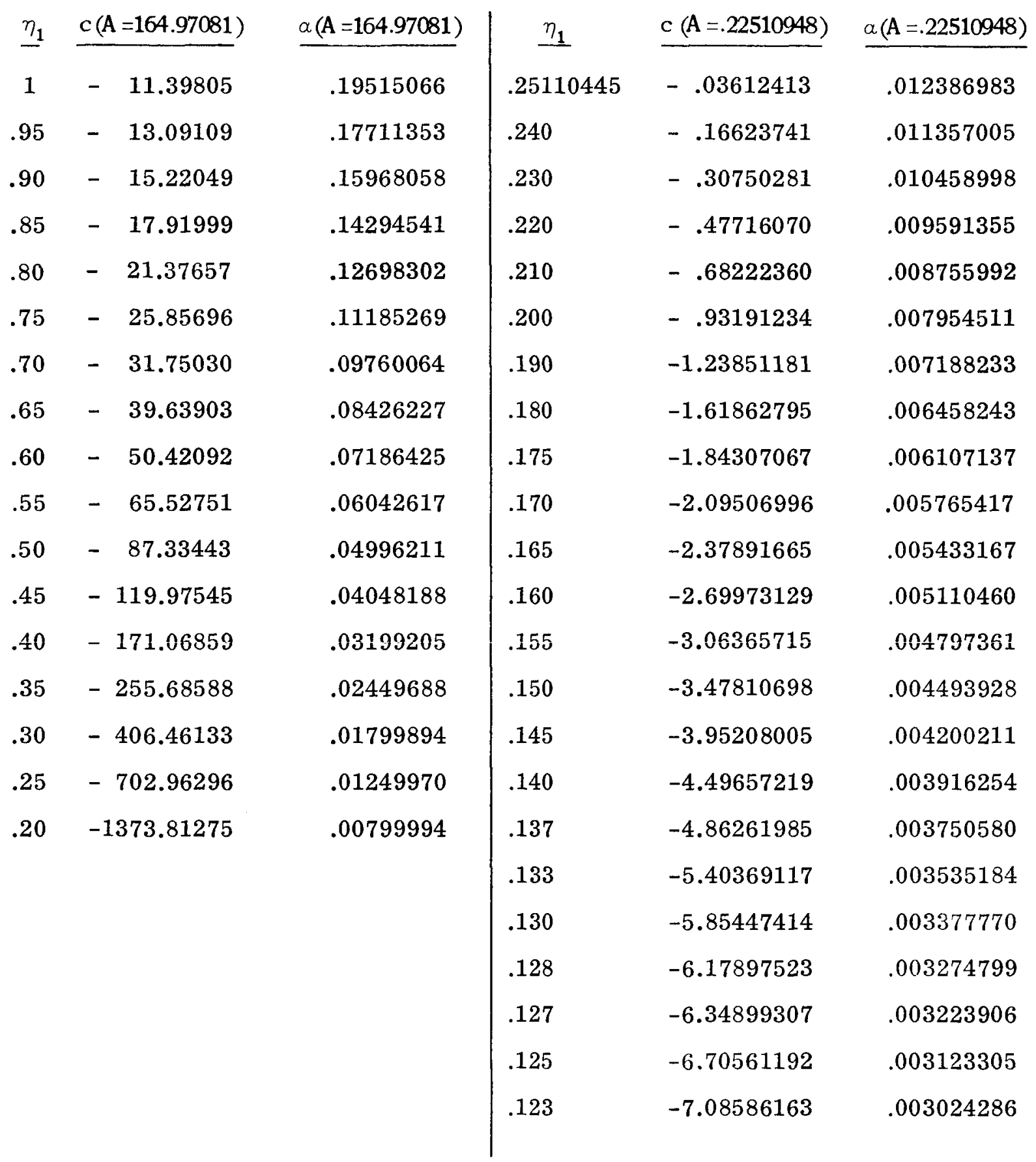


APPENDIX D

Graphs 


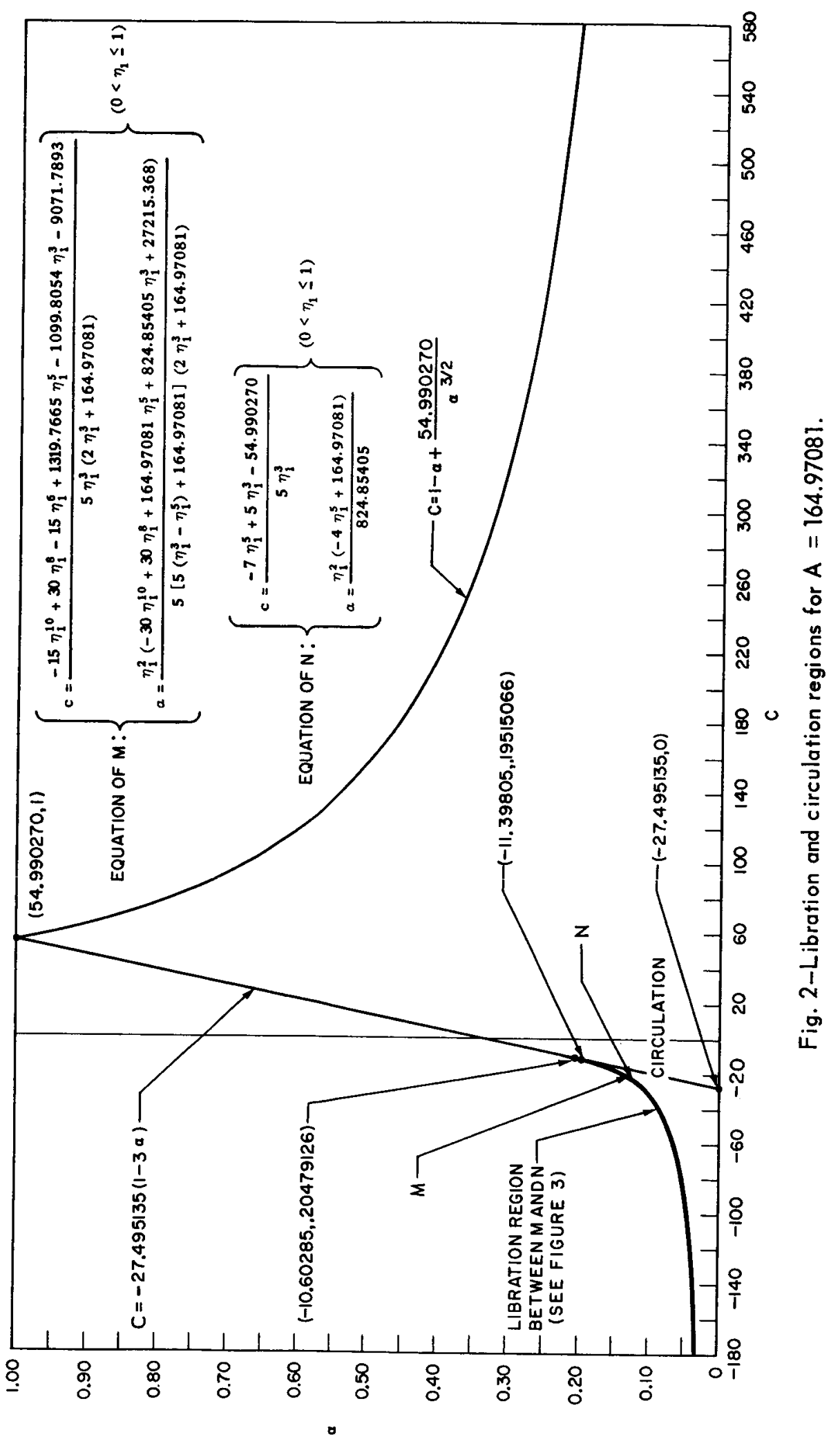



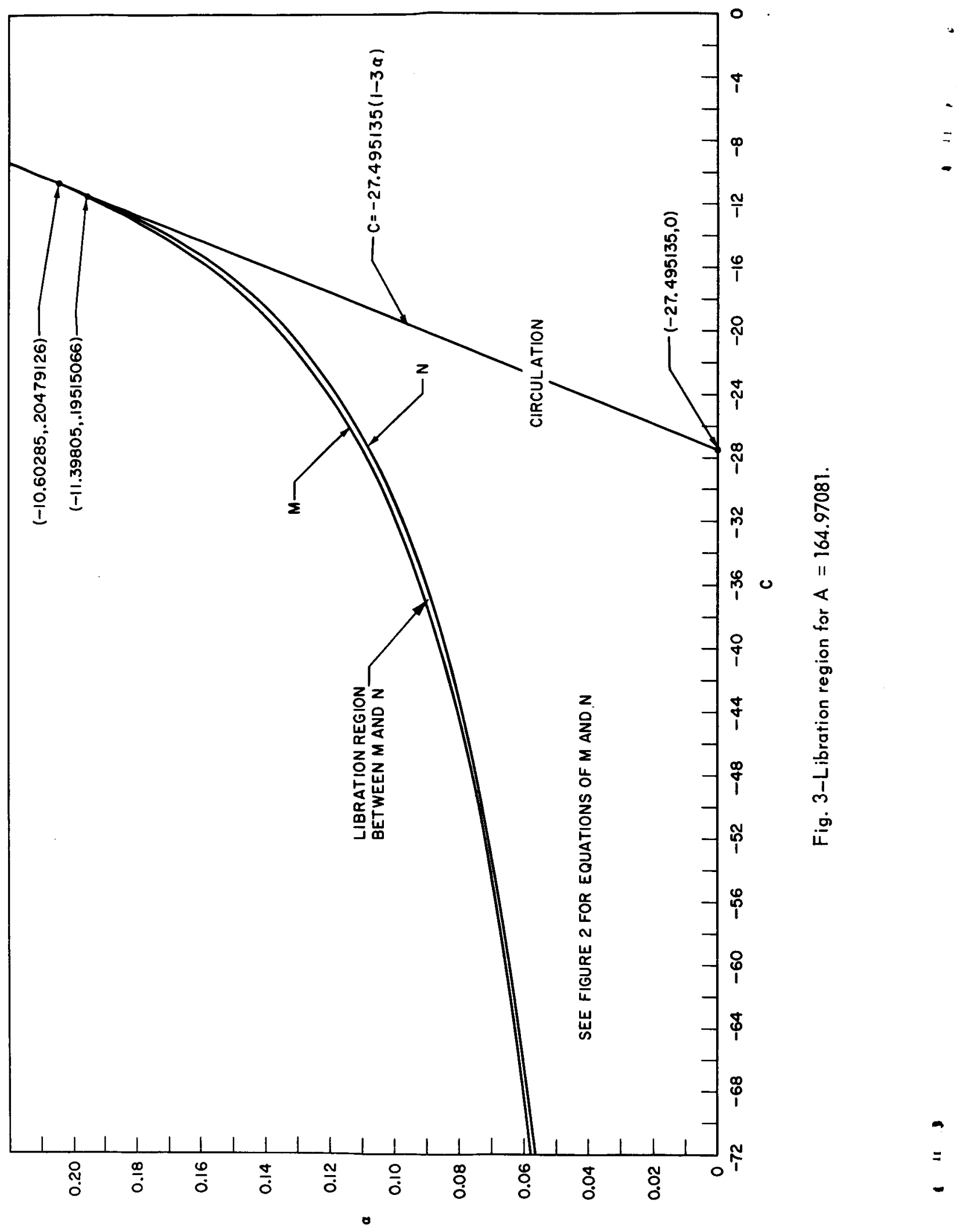


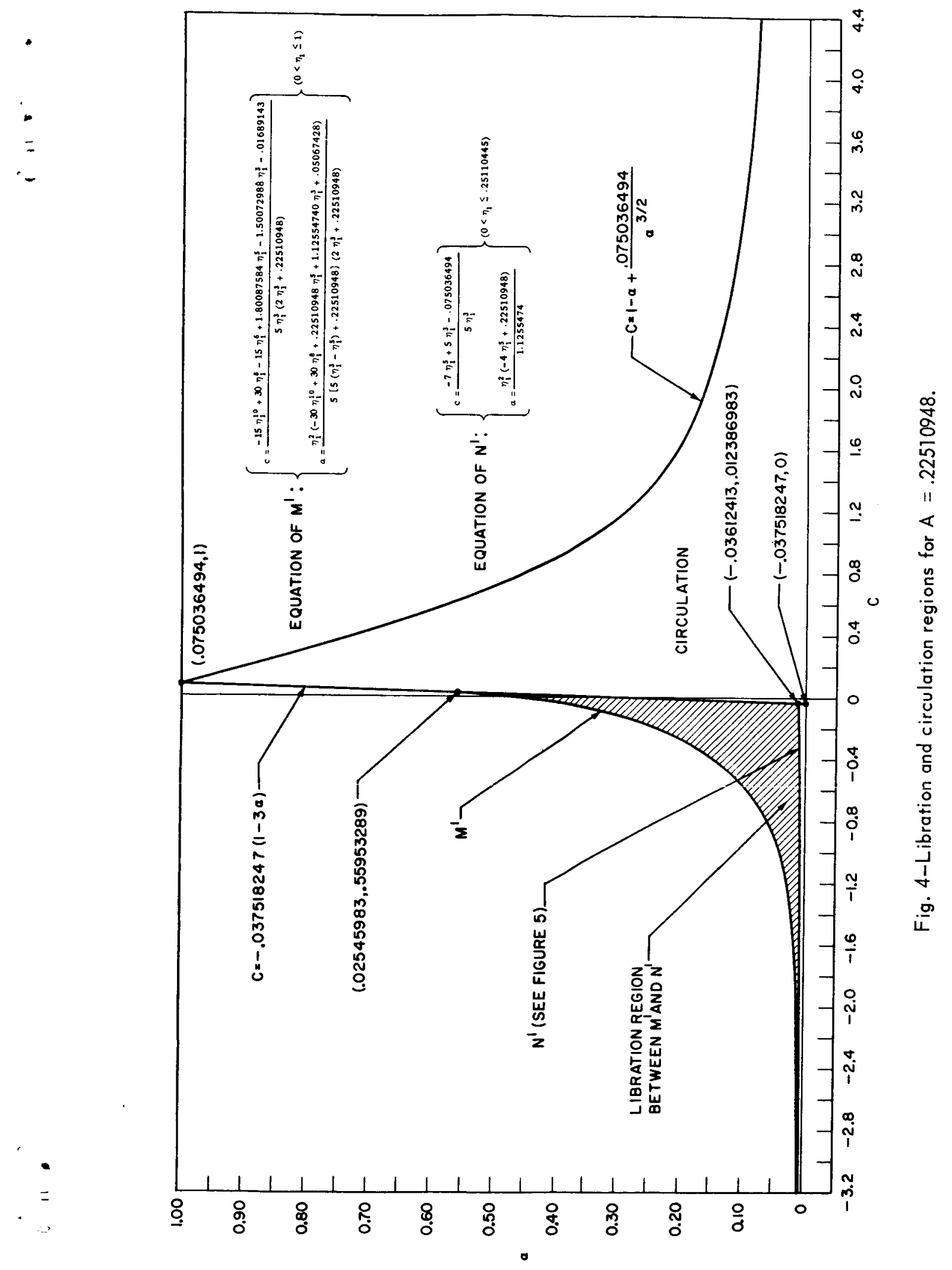



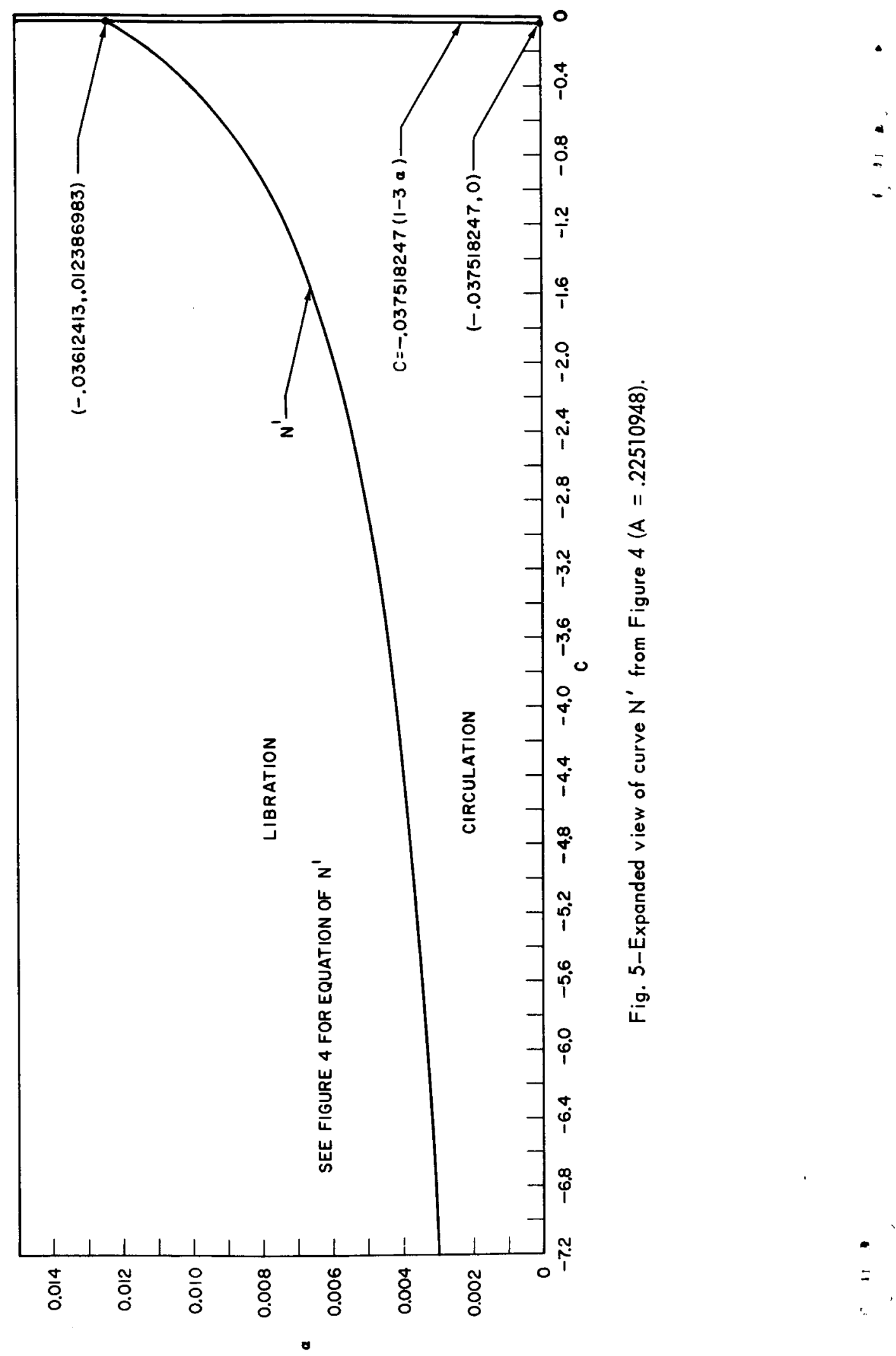